Abstract P23 Table 1 ELF and TE results for selected fibrosis stages

\begin{tabular}{|c|c|c|c|c|c|}
\hline \multirow[b]{2}{*}{ Fibrosis stage } & \multicolumn{2}{|l|}{ ELF score } & \multicolumn{2}{|l|}{ TE (kPa) } & \multirow[b]{2}{*}{ p Value* } \\
\hline & Median (IOR) & AUROC (95\% CI) & Median (IQR) & AUROC $(95 \%$ CI) & \\
\hline 0 vs $1-6$ & $8.1(0.8)$ vs $9.3(1.8)$ & $0.812(0.695$ to 0.930$)$ & $5.6(3.1)$ vs $8.4(5.4)$ & 0.798 (0.640 to 0.9257 & 0.826 \\
\hline $0-2$ vs $3-6$ & $8.4(1.1)$ vs 9.9 (1.5) & $0.831(0.771$ to 0.890$)$ & $6.5(2.6)$ vs $10.1(6.7)$ & $0.865(0.814$ to 0.916$)$ & 0.306 \\
\hline $0-4$ vs 5,6 & $9.0(1.4)$ vs $10.6(2.0)$ & 0.844 (0.777 to 0.912$)$ & $7.3(2.8)$ vs $15.4(11.7)$ & $0.946(0.911$ to 0.982$)$ & 0.003 \\
\hline
\end{tabular}

${ }^{*}$ Significance of comparison of ELF and TE AUROC.

predict clinical outcomes in a range of chronic liver diseases. This is the first study of performance of ELF in patients with chronic hepatitis $\mathrm{B}(\mathrm{CHB})$ using a single aetiology cohort, and we compare its performance with an alternative method of assessing fibrosis, transient elastography (TE).

Aim We aimed to compare the performance of ELF with transient elastography (TE) in a cohort of patients with $\mathrm{CHB}$, in the identification of liver fibrosis using liver biopsy as the reference standard. Method 188 patients with $\mathrm{CHB}$ were recruited consecutively at a single Italian centre. TE, serum sampling and liver biopsy were performed on the same day. ELF tests were performed in one batch at a central laboratory using thawed samples previously stored at $-20^{\circ} \mathrm{C}$. Biopsies were assessed by one pathologist using the Ishak staging system. Diagnostic performance of ELF and TE for detection of histological stages of liver fibrosis was assessed and area under receiver operator characteristic curves (AUROC) calculated. Only those in whom TE was successfully performed were included. Different fibrosis levels were assessed, from any fibrosis (0 vs $1-6)$ to cirrhosis ( $0-4$ vs 5,6 and $0-5$ vs 6$)$.

Results Patients were treatment-naive, median age was 47 years. $78 \%$ were e-antigen negative. Biopsies reported mild/moderate fibrosis (Ishak 0-1) in 21\%, moderate fibrosis (2-3) 41\% and severe fibrosis/ cirrhosis (4-6) 38\%. ELF and TE demonstrated good performance in identifying fibrosis (Abstract P23 table 1-representative fibrosis stages are shown, further data available). Both modalities showed similar performance in identifying any fibrosis and minimal fibrosis, with TE performing better in identifying moderate and severe fibrosis, and cirrhosis.

Conclusion In untreated patients with $\mathrm{CHB}$ with moderate and severe fibrosis or cirrhosis, TE correlated more closely with histological staging than ELF. The relatively modest performance of ELF in detecting severe fibrosis compared to previous studies may be attributable to disease aetiology or prolonged sample storage at $-20^{\circ} \mathrm{C}$. Further validation of TE and ELF in CHB should include analysis at the time of sampling and evaluation of the prognostic performance for clinical outcomes as well as histology.

\section{P24 PRESENCE OF IMPAIRED BARORECEPTOR SENSITIVITY IS A POOR PROGNOSTIC MARKER IN CIRRHOSIS}

doi:10.1136/gutjnl-2011-300857a.24

K Rye, G Mortimore, A Austin, J Freeman. Royal Derby Hospitals

Introduction Autonomic function is essential for blood pressure control and baroreceptor sensitivity (BRS) acts as a composite marker of overall function. Both sympathetic and parasympathetic function is impaired in cirrhosis. Impaired BRS predicts death in cardiovascular diseases and chronic kidney disease.

Aim The aim of the present study was to assess the relationship between BRS and liver disease severity, systemic and portal haemodynamics and mortality in cirrhosis.

Method Prospective study of 29 cirrhotic patients. Systemic haemodynamics and BRS were assessed non-invasively using the Finometer. ${ }^{\circledR}$ Spontaneous BRS was calculated from the regression of pulse interval on systolic blood pressure. Portal pressure was assessed by measurement of the hepatic venous pressure gradient (HVPG). Gastroscopy assessed variceal size and 1-year probability of bleeding according to the NIEC index.

Results 20 male (69\%), median age 47 years (42-55), Child-Pugh score 6 (Class A 18, B 10, C 1) and MELD 10 (8-13). BRS was impaired in cirrhosis (median $4.2 \mathrm{~ms} / \mathrm{mm} \mathrm{Hg}$, IOR 2.5-6.2 ms/ $\mathrm{mm} \mathrm{Hg}$ ) but was not related to Child-Pugh score or MELD. Significant differences in BRS were seen with respect to gender (Female $2.0 \mathrm{~ms} / \mathrm{mm} \mathrm{Hg}$ vs male $5.8 \mathrm{~ms} / \mathrm{mm} \mathrm{Hg}, \mathrm{p}=0.0125$ ), presence of varices (present $3.8 \mathrm{~ms} / \mathrm{mm} \mathrm{Hg}$ vs absent $8.3 \mathrm{~ms} / \mathrm{mm} \mathrm{Hg}$, $\mathrm{p}=0.0206$ ), and ascites (ascites $2.0 \mathrm{~ms} / \mathrm{mm} \mathrm{Hg}$ vs no ascites $5.3 \mathrm{~ms} /$ $\mathrm{mm} \mathrm{Hg}, \mathrm{p}=0.0433$ ). No significant differences in BRS were seen according to alcohol intake. A significant negative correlation was seen between BRS and age $(r=-0.46, p=0.0130)$, heart rate $(\mathrm{r}=-0.56, \mathrm{p}=0.0015)$, HVPG $(\mathrm{r}=-0.69, \mathrm{p}<0.0001)$ and 1-year probability of variceal bleeding $(\mathrm{r}=-0.43, \mathrm{p}=0.0199)$. Over a median follow-up of 710 days, $8 / 29(27.6 \%)$ patients died with median time to death 854 days. Mortality was significantly higher in patients with HVPG greater than or equal to $10 \mathrm{~mm} \mathrm{Hg}$ than without clinically significant portal hypertension $(p=0.0024)$ and with a MELD score greater than or equal to 17 ( $p<0.0001)$. When stratified according to BRS (less than, or greater than or equal to $6 \mathrm{~ms} / \mathrm{mm} \mathrm{Hg}$ ), mortality was significantly higher in patients with more severe impairment $(<6 \mathrm{~ms} / \mathrm{mm} \mathrm{Hg}$ ) than with preserved BRS (33\% vs $12.5 \%$, $\mathrm{p}=0.0034)$ despite no significant difference in Child-Pugh score, MELD, systemic haemodynamics, HVPG or length of follow-up. Patients with impaired BRS had significantly higher probability of variceal bleeding, and were more likely to have ascites ( $24 \%$ vs $0 \%$ ).

Conclusion BRS is not related to liver disease severity as assessed by Child-Pugh or MELD but a significant inverse correlation exists between BRS and HVPG. Compared to patients with preserved BRS, patients with significant impairment of BRS have a higher mortality, a higher probability of variceal bleeding and are more likely to have ascites. Impairment of BRS appears to be a poor prognostic factor independent of liver disease severity or portal pressure, possibly relating to a failure to respond to the haemodynamic challenges associated with complications in cirrhosis.

\section{P25 FRAGILITY FRACTURE RISK IN CIRRHOSIS: A COMPARISON OF THE FRACTURE RISK ASSESSMENT TOOL (FRAX), BSG AND NICE GUIDELINES}

doi:10.1136/gutjnl-2011-300857a.25

L Ayres, J Digby-Bell, S Clarke, A Dhanda, K Caddick, P Collins. University Hospital Bristol

Introduction Low bone mineral density (BMD) is common in patients with chronic liver disease, and tends to be related to the severity rather than aetiology of cirrhosis. BMD alone correlates modestly with fragility fracture. The BSG recommend DXA scanning all cirrhotic patients. NICE guidelines apply only to postmenopausal women.

The FRAX ${ }^{\circledR}$ score is a web-based tool incorporating clinical risk factors to predict the risk of osteoporotic fracture. Risk may be estimated without BMD, and refined with DXA. Pre-BMD FRAX ${ }^{\circledR}$ 
Abstract P25 Table 1 Patients recommended treatment vary according to guideline

\begin{tabular}{|c|c|c|c|c|}
\hline & $\begin{array}{l}\text { Pre-BMD } \\
\text { FRAX }{ }^{\circledR} \text { (NOGG) }\end{array}$ & $\begin{array}{l}\text { Post- BMD } \\
\text { FRAX }{ }^{\circledR} \text { (NOGG) }\end{array}$ & BSG & NICE \\
\hline Number of relevant patients & 103 & 103 & 132 & 43 \\
\hline Patients requiring DXA & 32 & NA & 132 & NA \\
\hline \multicolumn{5}{|c|}{ Anti-osteoporotic treatment recommended } \\
\hline Total & 9 & 16 & 25 & 3 \\
\hline High risk (FRAX® $+B M D)$ & 8 & 16 & 9 & 1 \\
\hline Low risk (FRAX $®+B M D)$ & 1 & $\mathrm{~N} / \mathrm{A}$ & 16 & 2 \\
\hline \multicolumn{5}{|l|}{ Treatment not recommended } \\
\hline Total & 94 & 87 & 107 & 40 \\
\hline High risk $\left(F R A X\left({ }^{\circledR}+B M D\right)\right.$ & 8 & $\mathrm{~N} / \mathrm{A}$ & 11 & 4 \\
\hline Low risk (FRAX $\left({ }^{\circ}+B M D\right)$ & 86 & 87 & 96 & 36 \\
\hline
\end{tabular}

categorises patients to low, intermediate or high risk according to set thresholds by the National Osteoporosis Guidelines Group (NOGG) and recommend lifestyle-advice, DXA or anti-osteoporosis treatment respectively (intermediate patients are rescored post-BMD to either high or low risk). Cirrhosis contributes to calculated fracture risk.

Aim To determine what proportion of our patients would be eligible for DXA and recommended treatment under BSG, NICE and $\mathrm{FRAX}^{\circledR} /$ NOGG guidelines. To calculate the correlation of the pre-BMD FRAX ${ }^{\circledR}$ scores with the post-BMD FRAX ${ }^{\circledR}$ scores.

Method We interrogated our outpatient database of cirrhotic patients and calculated the FRAX ${ }^{\circledR}$ score pre and post-BMD measurement. For each guideline we calculated the number of: patients eligible for treatment; high risk patients left untreated and low risk patients unnecessarily treated.

Results Of 132 patients $(58 \%$ male) with cirrhosis (mixed aetiology), 43 had normal $\mathrm{BMD}, 64$ were osteopaenic and 25 osteoporotic. 103/132 patients (43 postmenopausal women and 60 men $>50$ years) were eligible for FRAX ${ }^{\circledR}$. Of these, 64/103 were estimated low, 33/103 intermediate, and 6/103 at high risk of fracture. Of those at low risk (and therefore not required to undergo DXA scanning (NOGG)), 8 were reclassified as high risk post-BMD (one patient was reclassified from high to low risk). Patients eligible for treatment: pre-BMD FRAX ${ }^{\circledR} /$ NOGG 10/103, post-BMD FRAX ${ }^{\circledR}$ / NOGG 17/103, BSG 25/132, NICE 3/45. Low risk patients unnecessarily treated: pre-BMD FRAX ${ }^{\circledR} /$ NOGG 1 , BSG 16, NICE 2. High risk patients not recommended to receive treatment: 8,11 , and 4 . Pre-BMD FRAX ${ }^{\circledR}$ correlates well with post-BMD FRAX: Spearman's coefficient $r=0.76$, sensitivity of $47.5 \%$, specificity $98.8 \%$, PPV $90 \%$ and NPV $89 \%$ for correctly risk stratifying patients.

Conclusion BSG guidance does not incorporate clinical risks and is likely to expose some patients to unnecessary tests, or treatment. NICE is not widely applicable and does not suggest when to DXA. Cirrhosis is not listed as a risk factor, thus underestimating fracture risk. FRAX ${ }^{\circledR} /$ NOGG accurately identifies high risk patients and which group to DXA. Scanning only intermediate risk patients saves unnecessary scans, but in our cohort fails to identify 8/94 (8.5\%)

Abstract P25 Table 2 Comparison of pre-BMD and postBMD FRAX

\begin{tabular}{lrlc}
\hline & & \multicolumn{2}{l}{ Post-BMD FRAX } \\
\cline { 3 - 4 } Pre-BMD FRAX $^{\circledR}$ & & Low risk & High risk \\
\hline Low risk & 65 & 57 & 8 \\
Intermediate risk & 32 & 29 & 3 \\
High risk & 6 & 1 & 5 \\
Total & 103 & 87 & 16 \\
\hline
\end{tabular}

Abstract P25 Table 3 Prevalence of clinical risk factors

\begin{tabular}{lll}
\hline Clinical risk factors & $\begin{array}{l}\text { Males } \\
\mathbf{n = 7 6}(\%)\end{array}$ & $\begin{array}{l}\text { Female } \\
\mathbf{n = 5 6}(\%)\end{array}$ \\
\hline 0 & 0 & 0 \\
$\geq 1$ & $31(41)$ & $23(41)$ \\
$\geq 2$ & $45(59)$ & $33(59)$ \\
$\geq 3$ & $10(13)$ & $8(14)$ \\
$\geq 4$ & $1(1.3)$ & 0 \\
\hline
\end{tabular}

high risk patients. Male smokers with alcoholic cirrhosis were overrepresented in this group. Similarly to other cohorts, Pre-BMD FRAX $^{\circledR}$ has a low sensitivity but is highly specific when compared to post-FRAX ${ }^{\circledR}$ BMD.

\section{P26 RAPID IDENTIFICATION, TRIAGE AND MANAGEMENT OF ALCOHOL RELATED HOSPITAL ADMISSIONS}

doi:10.1136/gutjnl-2011-300857a.26

${ }^{1} \mathrm{R}$ J Aspinall, ${ }^{2} \mathrm{~S}$ Atkins, ${ }^{2} \mathrm{~K}$ Rackham, ${ }^{2} \mathrm{~T}$ Barratt, ${ }^{3} \mathrm{P}$ Schmidt, ${ }^{4} \mathrm{~J}$ Ward, ${ }^{2}$ Donna Bowman. ${ }^{1}$ Department of Gastroenterology and Hepatology, Queen Alexandra Hospital, Portsmouth, UK; ${ }^{2}$ Alcohol Liaison Service, Queen Alexandra Hospital, Portsmouth, UK; ${ }^{3}$ Medical Admissions Unit, Queen Alexandra Hospital, Portsmouth, UK; ${ }^{4}$ WMC Limited

Introduction Portsmouth Hospitals NHS Trust has the highest rate of alcohol related hospital admissions in the South East of England (1993 per 100000 population for 2009/2010). Admissions with complications of cirrhosis have doubled in under 4 years and recorded deaths from liver cirrhosis exceed the regional and national averages. As part of a comprehensive strategy to tackle the rising burden of alcoholic cirrhosis, a dedicated team of alcohol nurse specialists has been deployed to identify all alcohol related admissions at the earliest time point and initiate management. We conducted a prospective evaluation of the patients referred to the service over a 4-month period.

Aim To determine the demographic pattern of patients admitted with alcohol related illnesses in a single large hospital serving a population of 650000 .

Method All patients at the Queen Alexandra Hospital with an alcohol related admission identified during the study period were referred to the specialist nursing service and recorded on a secure database. A comprehensive clinical assessment was made by a team of 3 specialist nurses working to agreed protocols. The WHO Alcohol Use Disorders Identification Test (AUDIT) score was recorded along with data regarding age of first alcohol exposure, regular usage and problem drinking. Liver related morbidity was determined by clinical assessment, standard blood parameters and by calculation of Model for Endstage Liver Disease (MELD) and Glasgow Alcoholic Hepatitis (GAHS) scores.

Results During the study period, 495 new patients were referred to the service. $71 \%$ were identified and referred in the ED or MAU, the remainder from inpatient wards. Mean age was 50 years (range 18-91) and $72 \%$ were male. $97.5 \%$ of patients were of white British ethnicity and only $11 \%$ were in regular employment. There were no gender differences in the proportion of younger drinkers $<40$ years $(20 \%$ in both sexes). Overall, $12 \%$ of patients referred with alcohol misuse disorders were aged over 70 years. Our cohort demonstrated heavy current alcohol misuse with a mean weekly consumption of 229 units at admission (range 8-860) and $50 \%$ of patients recorded the maximum AUDIT score of 40 . The mean age of first drinking alcohol was 11 years (range 3-26) and the mean age of establishing regular use was 22 (range 11-65). Laboratory 